\title{
Determination of fracture mechanics parameters on a base of local displacement measurements
}

\author{
Yu. G. Matvienko \\ Mechanical Engineering Research Institute of the Russian Academy of Science (IMASH RAN). 4 M. Kharitonievsky Per., \\ 101990 Moscow, Russia.E-mail:matvienko7@yahoo.com.
}

V.S. Pisarev, S. I. Eleonsky

Central Aero-Hydrodynamics Institute named after Prof. N.E. Zhukovsky (Ts AGI). 1 Zhukovsky Street, Zhukovsky 140180 Moscow Region, Russia.E-mail:VSP5335@mail.ru.

\begin{abstract}
New experimental technique for a determination of the stress intensity factor (SIF) and T-stress values is developed and verified. The approach assumes combining the crack compliance method and optical interferometric measurements of local deformation response on small crack length increment. Initial experimental information has a form of in-plane displacement component values, which are measured by electronic speckle-pattern interferometry at some specific points located near a crack tip. Required values of fracture mechanics parameters follow from the first four coefficients of Williams' series. A determination of initial experimental data at the nearest vicinity of notch tip is the main feature of the developed approach. That is why it is not necessary to involve complex numerical models, which include global geometrical parameters, loading and boundary conditions of the object under study, in a stage of experimental data interpretation. An availability of high-quality interference fringe patterns, which are free from rigid-body motions, serves as a reliable indicator of real stress state around a crack tip. A verification of the technique is performed by comparing experimental results with analogous data of FEM modelling. Experimentally determined mode I SIF for DCB specimen with end crack is in 5 per cent agreement with the numerically simulated case. Proposed approach is capable of estimating an influence of the notch radius on fracture mechanics parameters. Comparing SIF and T-stress obtained for U-notches of different radius both in actual and residual stress field confirms this statement.
\end{abstract}

KEYWORDS. Stress intensity factor; T-stress; crack compliance method; In-plane displacement components; Electronic speckle-pattern interferometry.

\section{INTRODUCTION}

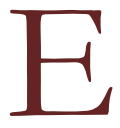
xperimental determination of stress intensity factor (SIF) and T-stress for a crack of constant length under external load increment is of considerable current interest [1-8]. At the same time the crack compliance method is capable of SIF deriving by local crack length increasing under constant load conditions [9-10]. This presentation is devoted to a development and verification of new technique for a determination of SIF and T-stress values by combining the crack compliance method and optical interferometric measurements of local deformation response on small crack length increment. 


\section{MAIN PRINCIPALS AND RELATIONS}

$\mathrm{M}$ odified version of the crack compliance method resides in recording interference fringe patterns, which correspond to a difference between two in-plane displacement component fields. Each field is referred to a crack of close but different length. The first exposure is made for a crack of initial length $a_{n-1}$ (see Fig. 1). Then initial crack length is increased by small increment $\Delta a_{n}$ so that new total crack length becomes equal to $a_{n}=a_{n-1}+\Delta a_{n}$ and the second exposure is performed. Required interference fringe patterns are visualized by numerical subtraction of two images recorded for two cracks [11]. Two interferograms, which are obtained by this way for thin plate with through edge crack of mode I, are shown in Fig. 2. Positive direction of $x$-axis in Fig. 1 and Fig. 2 coincides with a direction of the crack propagation.

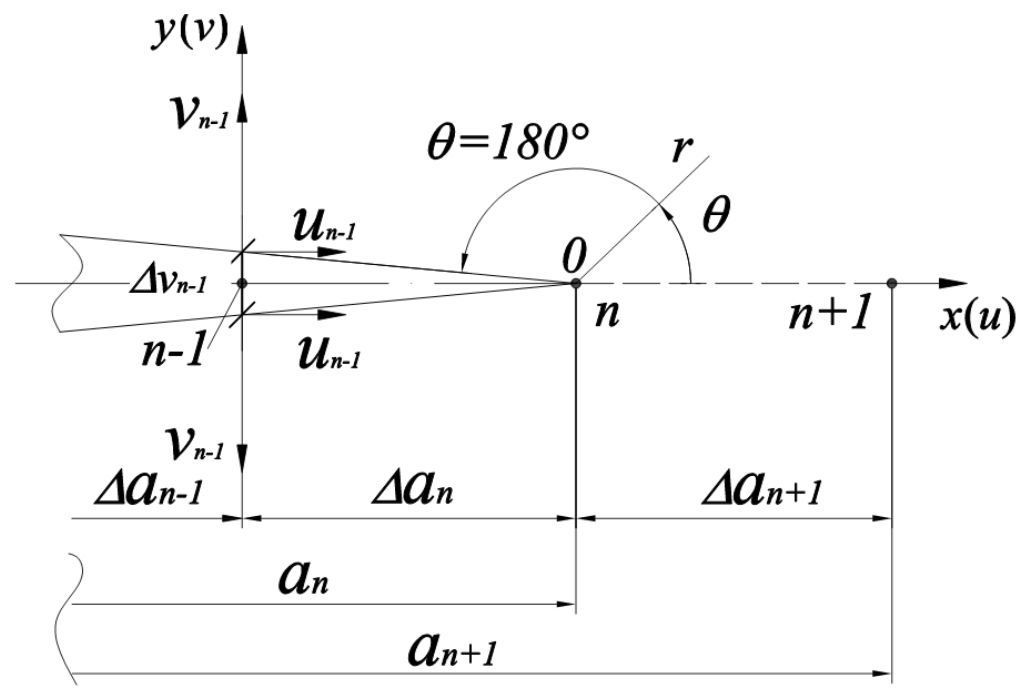

Figure 1: Polar co-ordinate system related to a crack tip and the notation adopted.

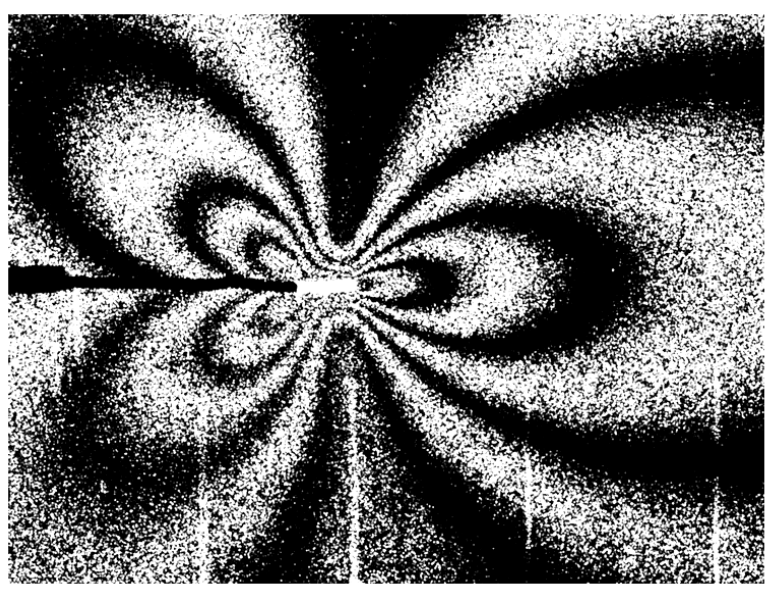

(a)

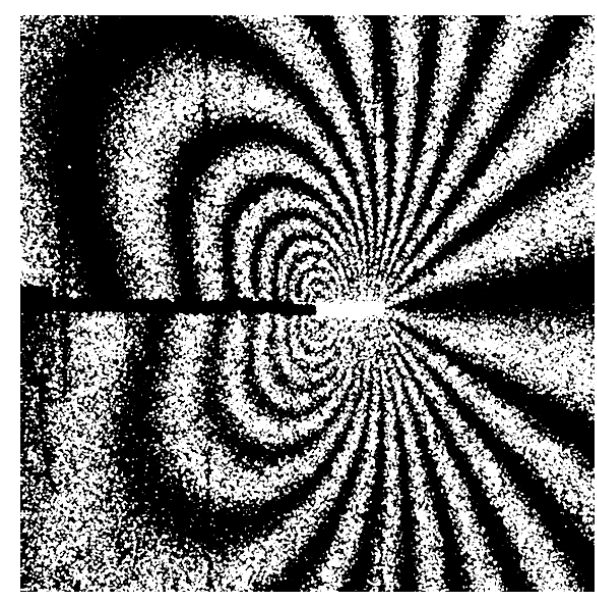

(b)

Figure 2: Specimen \#3V. Interference fringe pattern obtained in terms of in-plane displacement component $u$ (a) and $v$ (b). Initial crack length $a_{4}=7.18 \mathrm{~mm}$ with increment $\Delta a_{5}=1.81 \mathrm{~mm}$

Procedure of deriving required fracture mechanics parameters from interference fringe patterns is based on Williams' formulation [12]. In-plane displacement field near a crack tip is expressed as an infinite series for each in-plane displacement component. When $x$-direction coincides with the crack line these series for mode I condition have the following form: 


$$
\begin{aligned}
& u=\sum_{n=1}^{\infty} \frac{r^{\frac{n}{2}}(1+\mu)}{E} A_{n}\left\{\left[k+\frac{n}{2}+(-1)^{n}\right] \cos \frac{n \theta}{2}-\frac{n}{2} \cos \frac{(n-4) \theta}{2}\right\} \\
& v=\sum_{n=1}^{\infty} \frac{r^{\frac{n}{2}}(1+\mu)}{E} A_{n}\left\{\left[k-\frac{n}{2}-(-1)^{n}\right] \sin \frac{n \theta}{2}-\frac{n}{2} \sin \frac{(n-4) \theta}{2}\right\}
\end{aligned}
$$

where $u$ and $v$ are in-plane displacement component in direction of $x$ and $y$ axis, respectively; $\mu$ is the Poisson's ratio; $E$ is the elasticity modulus; $k=(3-\mu) /(1+\mu)$ for plane stress and $k=(3-4 \mu)$ for plane strain conditions; $A_{n}$ are constants to be determined; $r$ and $\theta$ are radial and angular distance from the crack tip as it is shown in Fig. 1.

Values of stress intensity factor (SIF) $K_{I}$ and T-stress $T$ are connected with coefficients of infinite series (1) by the following way [7]:

$$
K_{I}=A_{1} \sqrt{2 \pi}, T=4 A_{2}
$$

Generally initial experimental information represents a difference in absolute values of in-plane displacement components $U_{n}(r, \theta)$ and $V_{n}(r, \theta)$ for two cracks of length $a_{n}$ and $a_{n-1}$ :

$$
U_{n}(r, \theta)=u_{n}(r, \theta)-u_{n-1}(r, \theta), V_{n}=v_{n}(r, \theta)-v_{n-1}(r, \theta)
$$

where $u_{n-1}(r, \theta), v_{n-1}(r, \theta)$ and $u_{n}(r, \theta), v_{n}(r, \theta)$ are absolute values of in-plane displacement components in a point with polar co-ordinates $(r, \theta)$ for a crack of $a_{n-1}$ and $a_{n}$ length, respectively.

Eq. (3) are valid for any point belonging to the proximity of crack tip located at point $n$. But right hand sides of Eq. (3) include relative values of displacement components, which can not be directly used for a determination of $A_{n}$-values from decomposition (1). The key point of the developed approach resides in the fact that each interference fringe pattern of type shown in Fig. 2 contains a set of specific points located at a crack border immediately. Absolute values of in-plane displacement components and then coefficients $A_{n}$ from formulae (1) for a crack of $a_{n}$ length can be determined at these points.

First, specific points are located along the crack line between point $n-1$ and point $n$ where displacement component $v_{n-1}(r, \theta)$ equals to zero before making a crack length increment. Thus, interference fringe pattern shown in Fig. $2 \mathrm{~b}$ allows determining absolute values of $v_{n}(r, \theta)$-component for each point with polar co-ordinates $0 \leq r \leq \Delta a_{n}$ and $\theta=\pi$. Developed approach employs four first coefficients of series (1) for deriving required fracture mechanics parameters. A distribution of $v_{n}(r, \theta)$-displacement component along the crack line $(\theta=\pi$, see Fig. 1$)$ is expressed as:

$$
v_{n}(r, \theta=\pi)=\frac{4 \sqrt{r}}{E} A_{1}^{n}-\frac{4 r \sqrt{r}}{E} A_{3}^{n}+0(r)
$$

Relation (4) shows that deriving $K_{I}$ value from Eq. (3) demands a determination of $v_{n}(r, \theta)$-values at two points belonging to the interval $0 \leq r \leq \Delta a_{n}, \theta=\pi$, as minimum. It is conveniently to use two points with polar coordinates $\left(r=\Delta a_{n}, \theta=\pi\right)$ and $\left(r=\Delta a_{n} / 2, \theta=\pi\right)$. Substituting these co-ordinates into relation (4) forms a system of linear algebraic equations, a solution of which is:

$$
\begin{aligned}
& A_{1}^{n}=\frac{E}{8 \sqrt{\Delta a_{n}}}\left\{2 \sqrt{2} \Delta v_{n-0.5}-\Delta v_{n-1}\right\} \\
& A_{3}^{n}=-\frac{E}{4 \Delta a_{n} \sqrt{\Delta a_{n}}}\left\{\Delta v_{n-1}-\sqrt{2} \Delta v_{n-0.5}\right\}
\end{aligned}
$$

where $A^{n}{ }_{1}$ and $A^{n}{ }_{3}$ are coefficients of decomposition (1) for a crack of $a_{n}$ length; $\Delta v_{n-1}=2 v_{n}\left(r=\Delta a_{n}, \theta=\pi\right)$ and $\Delta v_{n-0.5}=$ $2 v_{n}\left(r=\Delta a_{n} / 2, \theta=\pi\right)$ are crack opening values from Eq. (4), which have to be experimentally determined. SIF value for a crack of $a_{n}$ length follows from combining the first relations from Eqs. (2) and (5):

$$
K_{I}^{n}=\frac{E \sqrt{2 \pi}}{8 \sqrt{\Delta a_{n}}}\left\{2 \sqrt{2} \Delta v_{n-0.5}-\Delta v_{n-1}\right\}
$$


Taking into account only the first term from Eq. (1) leads to well-known Westergaard relation:

$$
K_{I}^{n^{*}}=\frac{\Delta v_{n-1} E \sqrt{2 \pi}}{8 \sqrt{\Delta a_{n}}}
$$

A characterisation of $T$-stress values $T$ is based on a determination of $u$-displacement component directed along $x$-axis. A distribution of $u_{n}(r, \theta)$-displacement component for points belonging to the crack line $(\theta=\pi$, see Fig. 1), which corresponds to the second and the fourth terms of infinite series (1), is expressed as:

$$
u_{n}(r, \theta=\pi)=-\frac{4 r}{E} A_{3}^{n}+\frac{4 r^{2}}{E} A_{4}^{n}+0(r)
$$

Absolute value of $u$-component for a crack of $a_{n}$ length can be again obtained at point $n-1$ with polar co-ordinates $r=\Delta a_{n}$ and $\theta=\pi$ because at this point $u_{n-1}(r, \theta)=0$. A substitution of $r=\Delta a_{n}$ and $u_{n}\left(r=\Delta a_{n}, \theta=\pi\right)=u_{n-1}$ in Eq. (8) and taking into account the first from relations (3) lead to the following relation:

$$
u_{n-1}=-\frac{4 \Delta a_{n}}{E} A_{2}^{n}+\frac{4\left(\Delta a_{n}\right)^{2}}{E} A_{4}^{n}
$$

Eq. (9) gives us the first equation essential for a determination of T-stress value $T$ when displacement component value $u_{n}$ 1 is experimentally obtained. It should be noted that all experimental parameters needed for relations (5)-(7) and Eq. (9) can be derived from two interferograms, which correspond to $\Delta a_{n}$ crack length increment.

A formulation of the second required equation demands involving interference fringe pattern, which corresponds to crack length increasing from point $n$ to point $n+1$ by $\Delta a_{n+1}$ increment (see Fig. 1). For point $n+1$ with polar co-ordinates $\left(r=\Delta a_{n+1}, \theta=0\right)$ the first Eq. (4) can be written as:

$$
U_{n+1}=u_{n+1}\left(r=\Delta a_{n+1} ; \theta=0\right)-u_{n}\left(r=\Delta a_{n+1} ; \theta=0\right)=0-u_{n}\left(r=\Delta a_{n+1} ; \theta=0\right)=-u_{n+1}
$$

Combining relations (1) and (10) gives:

$$
-u_{n+1}=\frac{2 \sqrt{\Delta a_{n+1}}(1-\mu)}{E} A_{1}^{n}+\frac{4 \Delta a_{n+1}}{E} A_{2}^{n}+2 \Delta a_{n+1} \sqrt{\Delta a_{n+1}} \frac{(1-\mu)}{E} A_{3}^{n}+\frac{4\left(\Delta a_{n+1}\right)^{2}}{E} A_{4}^{n}
$$

where $u_{n+1}$ is the absolute value of $u_{n}(r, \theta)$ displacement component at point $n+1$ (see Fig. 1). Relation (11) represents the second equation essential for a determination of T-stress because the values of coefficients $A_{1}$ and $A_{3}$ are already known from formulae (5) Note that a value of $u_{n+1}$ has to be experimentally derived from interference fringe pattern of type shown in Fig. 2a, which are recorded for $\Delta a_{n+1}$ crack length increment. If an estimation of T-stress value is restricted by coefficient $A^{n}{ }_{2}$ only, the following simplified formula is valid:

$$
T^{n}=-\frac{u_{n}}{\Delta a_{n}} E
$$

The T-stress value in Eq. (12) can be determined by using interference fringe pattern of type shown in Fig. 2a recorded for crack length increment $\Delta a_{n}$ only.

Electronic speckle-pattern interferometry (ESPI) serves for a determination of in-plane displacement components [11]. Well-known optical system with normal illumination with respect to plane object surface and two symmetrical observation directions is used. When a projection of illumination directions onto plane surface of the investigated object coincides with $\xi$-direction, interference fringe pattern is described as:

$$
d_{\xi}=N \frac{\lambda}{2 \sin \Psi}
$$

where $d \xi$ is in-plane displacement component in $\xi$-direction; $N= \pm 1 ; \pm 2 ; \pm 3, \ldots$ are the absolute fringe orders; $\lambda=0.532$ $\mu \mathrm{m}$ is the wavelength of laser illumination; $\Psi=45$ degrees is the angle between inclined illumination and normal observation directions. When $\xi$-direction coincides with $x$-axis and $y$-axis displacement component $u$ and $v$ can be derived accordingly to formula (13), respectively. 


\section{METROLOGICAL VERIFICATION}

$\mathrm{M}$

etrological verification of the developed approach is performed by using specially designed specimen \#3V made from 2024 aluminium alloy $(E=74000 \mathrm{MPa}, \mu=0.33$ ) shown in Fig. 3. Working part of this specimen is a thin plate of dimensions $120 \times 48 \times 5 \mathrm{~mm}^{3}$. A U-notch of length $a_{0}=20 \mathrm{~mm}$ is initially made in the middle of the shortest side in a direction of the symmetry cross-section. The specimen is loaded by transverse force directed orthogonally to the notch line as it is shown in Fig. 3. Step-by-step notch increase process is firstly performed by narrow jewellery saw of width $b_{1}=0.3 \mathrm{~mm}$ (notch radius $\varrho_{1} \sim 0.15 \mathrm{~mm}$ ). A scheme of the experiment involved resides in the following. External transverse load $P_{n 1}$ is applied to the specimen. The first exposure is made for a notch of current length $a_{n-1}$. Then notch length is increased by small increment $\Delta a_{n}$ and the second exposure is made for a notch of the final length $a_{n}=a_{n-1}+\Delta a_{n}$. During a process of notch length increasing a value of acting force is slightly decreased to $P_{n 2}$ due to a compliance of the force gage. Two interference fringe patterns recorded for the $4^{\text {th }}$ notch length increment are shown in Fig. 2.

Interference fringe patterns are recorded for 7 notch length increments starting from initial notch length $a_{0}=20 \mathrm{~mm}$ up to $a_{0}+a_{n}=32.9 \mathrm{~mm}$. Experimental data are obtained for the same loading conditions: $P_{n 1}=0.930 \mathrm{kN}$ and $P_{n 2}=0.846 \mathrm{kN}$. Dependences of SIF $K_{I}$ and T-stress $T$ values from total notch length constructed accordingly to relation (6) and relations (9)-(11), respectively, are shown in Fig. 4. Note that SIF values obtained by formula (6) coincide with analogous data from formula (7) within 5 per cent because $A^{n}{ }_{3}$ values are practically equal to zero for all steps considered. This fact gives us a capability of reliable comparing experimentally obtained SIF values with analogous results of numerical simulation on a base of NASTRAN computer codes.
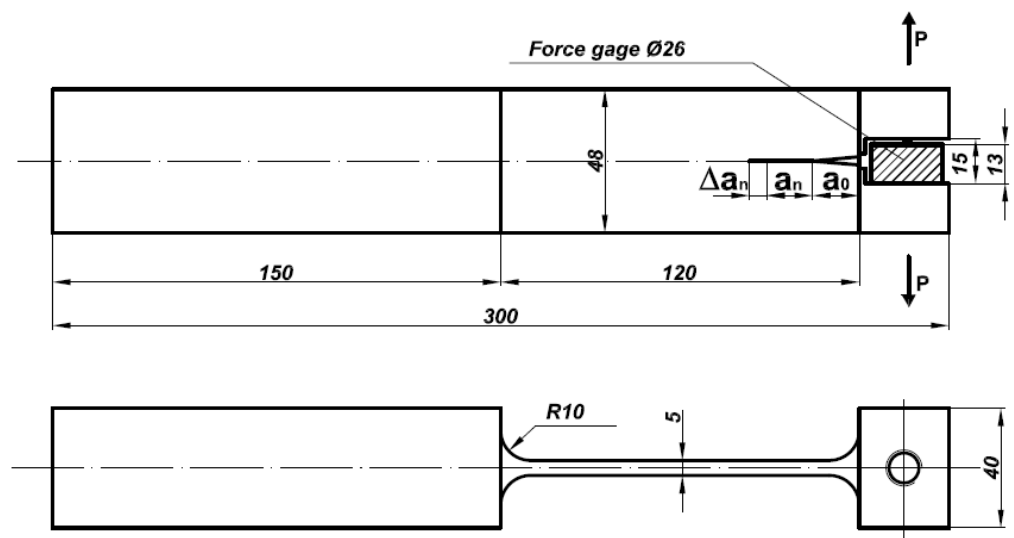

Figure 3: Drawing Specimen \#3V and a scheme of its loading.

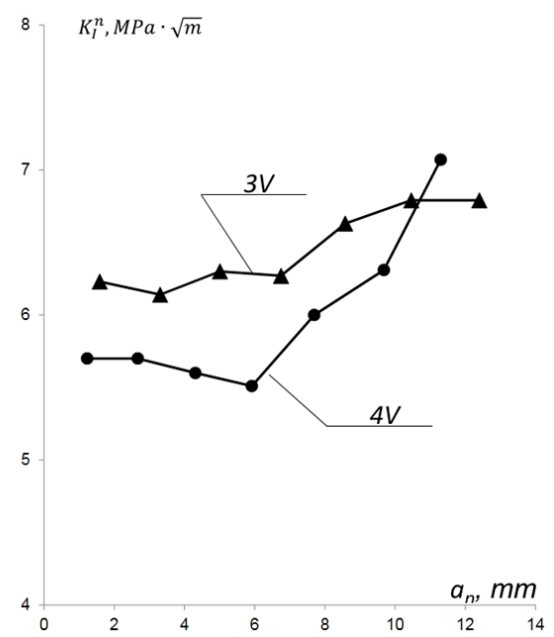

(a)

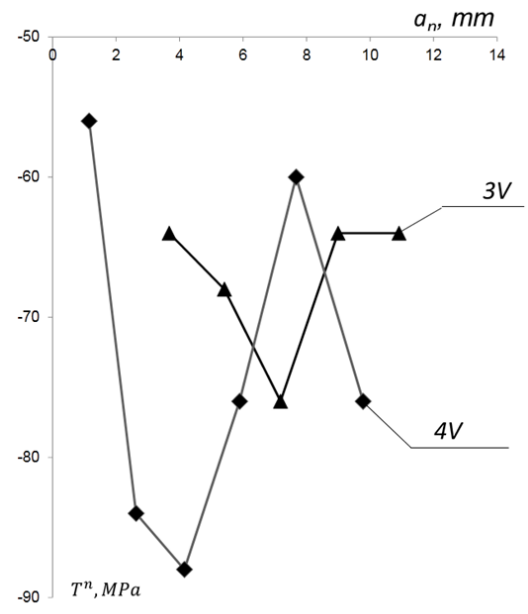

(b)

Figure 4: Dependencies of SIF $K_{I}(\mathrm{a})$ and T-stresses $T$ (b) from total notch length for specimen \#3V and specimen \#4V. 
Comparing the results of two types is performed for the fourth notch length increment with total notch length $a_{0}+a_{4}=$ $27.2 \mathrm{~mm}$. Finite element mesh consists of 162000 plane shell elements of CQUAD 4 type. Boundary conditions correspond to real geometry of the specimen shown in Fig. 3. Local area including a notch tip is simulated by twodimensional notch tip element CRAC2D. This element is capable of SIF calculation accordingly to formula (7) only. Numerical SIF value $K_{I}^{F E M}=6.48 M P a \sqrt{m}$ coincides with analogous experimental value $K_{I}^{4}=6.14 M P a \sqrt{m}$ within five per cent. This result proves a high accuracy and reliability of the developed approach.

\section{INFLUENCE OF THE NOTCH WIDTH ON FRACTURE MECHANICS PARAMETERS}

$\mathrm{D}$ eveloped approach is capable of determining fracture mechanics parameters for notches as well as cracks in both actual and residual stress field. It is also possible to estimate an influence of notch radius on SIF and T-stress values obtained through the use of formula (5) and relations (9)-(11), respectively. The first step in this way is made for actual stress field in specimen \#4V, geometrical parameters and loading conditions of which completely coincide with scheme shown in Fig. 3. The main difference resides in using a saw of width $b_{3}=1.0 \mathrm{~mm}$ (notch radius $\varrho_{3} \sim 0.50 \mathrm{~mm}$ ) for incremental crack length increasing. Dependences of SIF $K_{I}$ and T-stress $T$ values obtained accordingly to formula (6) and relations (9)-(11) from total notch length for specimen \#4V are also shown in Fig. 4. A difference in SIF values reaches 10 per cent with notch width increasing leads to SIF decreasing. Average T-stress values calculated for the first six steps are $T_{n}{ }^{a v}=-67.2 \mathrm{MPa}$ and $T_{n}{ }^{a v}=-76.8 \mathrm{MPa}$ for specimens \#3V and \#4V, respectively. Thus, notch width increasing gives a decrease of negative $T$-stress values by 12.5 per cent.

A study of notch increase in residual stress field is performed for two welded thin plates of dimensions $200 \mathrm{x} 100 \mathrm{x} 4 \mathrm{~mm} \mathrm{~m}^{3}$ made from aluminium alloy $(E=72000 \mathrm{MPa}, \mu=0.33)$. These plates are denoted as specimen $\# 015$ (notch width $b_{2}=0.6$ $\mathrm{mm}$, notch radius $\varrho_{2} \sim 0.30 \mathrm{~mm}$ ) and specimen \#016 (notch width $b_{1}=0.3 \mathrm{~mm}$, notch radius $\varrho_{1} \sim 0.15 \mathrm{~mm}$ ). Weld seam of lengths $100 \mathrm{~mm}$ coincides with one from symmetry cross-section of each specimen. Notches in both specimens are increased from a centre of the weld along the second symmetry cross-section orthogonally to the weld direction. Preliminary determination of maximal residual stress values $\sigma_{y}^{\max }$ acting along the weld in both specimens serves for estimating an identity of residual stress fields. These values determined at points with co-ordinate $x=9 \mathrm{~mm}$ equal to $\sigma_{y}^{\text {max }}$ $=130$ and $\sigma_{y}^{\max }=139 \mathrm{MPa}$ for Specimen \#015 and \#016, respectively. Data are obtained by combining the hole drilling method and ESPI. Holes are drilled in specimen's area, which does not contain a notch.

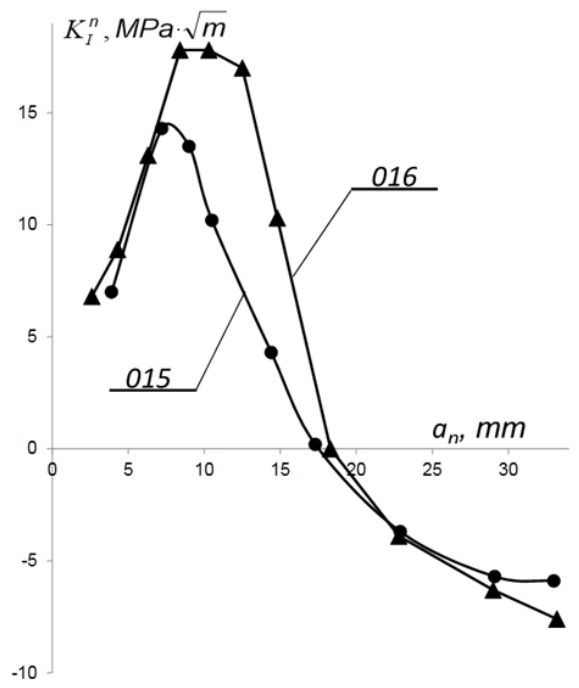

(a)

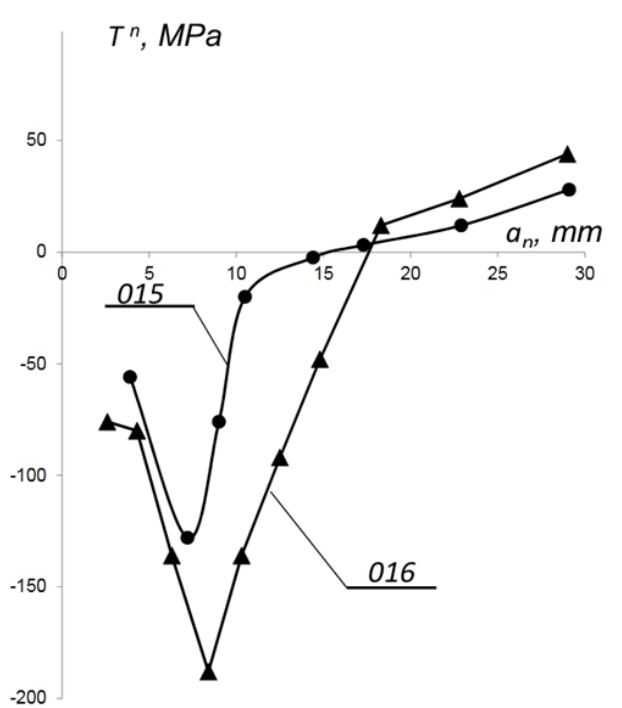

(b)

Figure 5: Dependences of SIF $K_{I}(\mathrm{a})$ and T-stress $T(\mathrm{~b})$ values form total notch length.

Experimental technique and a procedure of SIF and T-stress determination completely corresponds to the approach described above. Dependences of SIF $K_{I}$ and T-stress $T$ values from total notch length are shown in Fig. 5a and Fig. 5b, respectively. These results show that co-ordinate of points where $K_{I}=0$ and $T=0$ do not depend on the notch radius and correspond to notch length $a_{s}=16 \div 18 \mathrm{~mm}$. Maximal SIF values for specimen \#016 $\left(K_{I}=17.8 \mathrm{MPa}^{0} \mathrm{~m}^{0.5}\right)$ and $\mathrm{specimen}$ 
\#015 $\left(K_{I}=14.3 \mathrm{MPa} \cdot \mathrm{m}^{0.5}\right)$ differ by 20 per cent. A difference in maximal values of $\mathrm{T}$-stress for specimen \#016 $(T=-188$ $\mathrm{MPa}$ ) and specimen \#015 ( $T=-120 \mathrm{MPa})$ reaches 30 per cent. Distributions of $K_{I}$ in Fig. $5 \mathrm{a}$ are constructed by using formula (6). T-stresses shown in Fig. 5b are derived on a base of relations (9)-(11). It should be specially noted that formula (12) gives $T=0$ for both specimens and any notch length increment.

\section{CONCLUSIONS}

$\mathrm{N}$ ew technique for a determination of fracture mechanics parameters is developed. Its essence resides in a measurement of local deformation response on small crack length increment by electronic speckle-pattern interferometry. Obtained experimental information is capable of deriving the first four coefficients of Williams' asymptotic series and further calculations of stress intensity factor and T-stress values. Developed approach allows estimating dependencies of fracture mechanics parameters from a real width of the U-notch and/or notch radius.

\section{ACKNOWLEDGEMENT}

$\mathrm{P}$ resented study is performed in the framework of RFBR Grant \#10-08-00393-a and the Program of joint investigations of Central Aero-Hydrodynamics Institute and Mechanical Engineering Research Institute of the Russian Academy of Science.

\section{REFERENCES}

[1] Maleski, M.J., Kirugulige, M.S., H.V. Tippur, H.V., A method for measuring mode I crack tip constraint under static and dynamic loading conditions, Exp. Mech., 44 (2004) 522-532.

[2] Hild, F., Roux, S., Measuring stress intensity factors with a camera: Integrated digital image correlation (I-DIC), C. R. Mecanique, 334 (2006) 8-12.

[3] Yoneyama, S., Morimoto, Y., Takashi, M., Automatic evaluation of mixed-mode stress intensity factors utilizing digital image correlation, Strain, 42(2006) 21-29.

[4] Yoneyama, S., Ogawa, T., Kobayashi, Y., Evaluating mixed-mode stress intensity factors from full-field displacement fields obtained by optical methods, Eng. Fracture Mech., 74(2007) 1399-1412.

[5] Réthoré, J., Roux, S., Hild, F., Noise-robust stress intensity factor determination from kinematic field measurements, Eng. Fracture Mech., 75(2008) 3763-3781.

[6] López-Crespo, P., Burguete, R.L., Patterson, E.A., Shterenlikht, A., Withers, P.J., Yates, J.R., Study of a crack at a fastener hole by digital image correlation. Exp. Mech., 49(2009) 551-559.

[7] Yates, J.R., Zanganeh, M., Tai, Y.H., Quantifying crack tip displacement fields with DIC, Eng. Fracture Mech., 77(2010) 2063-2076.

[8] Hadj Meliani, M., Azari, Z., Pluvinage, G., Matvienko, Yu.G., The effective T-stress estimation and crack paths emanating from U-notches, Eng. Fracture Mech., 77(2010) 1682-1692.

[9] Schindler, H.-J., Determination of residual stress distributions from measured stress intensity factors. Int. J. of Fracture, 74(1995) R23-R30.

[10] Schindler, H.-J., Cheng, W., Finnie, I., Experimental determination of stress intensity factors due to residual stresses. Exp. Mech., 37(1997) 272-277.

[11] Rastogi, P., Digital speckle pattern interferometry and related techniques. Wiley, West Sussex, (2001).

[12] Williams, M.L., On the stress distribution at the base of a stationary crack. ASME J. of Applied Mech., 24(1957) 109114. 